González-Mayorga, H., Vieira, M.J. y Vidal, J. (2022). El uso de los resultados españoles de PISA en publicacionès científicas. Revista de Investigación Educativa, 40(1), 183-202.

DOI: http://dx.doi.org/10.6018/rie.451201

\title{
El uso de los resultados españoles de PISA en publicaciones científicas
}

\section{The use of the Spanish PISA results on scientific publications}

\author{
Héctor González-Mayorga, María-José Vieira' y Javier Vidal \\ Departamento de Psicología, Sociología y Filosofía. Facultad de Educación. Universidad de León (España)
}

\section{Resumen}

En la última década, el interés por los resultados de PISA ha crecido, tanto en el debate público como en el uso de los datos para la investigación. El objetivo de este estudio consiste en analizar la utilización de los resultados españoles de PISA por parte de la comunidad científica. Se realizó un análisis bibliométrico de 119 artículos científicos incluidos en las bases de datos Scopus, Eric EBSCOHost y Web of Science entre 2002 y 2019 mediante análisis de frecuencias, análisis MDS y regresiones logísticas binarias. Los resultados reflejan el creciente interés por los resultados españoles de PISA desde 2005 hasta la actualidad, perteneciendo los autores principalmente al ámbito universitario de la Educación. Respecto al uso de la información de PISA, la mayoría de los estudios emplean los datos como vía para generar conocimiento, siendo los temas socioeconómicos como la familia y la equidad los que han tenido una mayor presencia junto al rendimiento de los estudiantes. Se concluye que, a pesar de que PISA ha tardado en tener impacto entre la comunidad académica española, sus resultados son cada vez más utilizados como fuente de información primaria para la realización de estudios detallados sobre temas muy diversos. No obstante, a su vez, PISA se utiliza de forma genérica como indicador de calidad del sistema educativo, sin entrar en detalle en el análisis contextualizado del rendimiento en las competencias objeto de estudio.

Palabras clave: PISA; difusión de la información; evaluación normativa, competencias; análisis bibliométrico.

1 Correspondencia: María-José Vieira, maria.vieira@unileon.es, Departamento de Psicología, Sociología y Filosofía. Campus de Vegazana, S/N. CP. 24071. León, España. 


\begin{abstract}
In the last decade, interest in PISA results has grown, both in public debate and in the use of its data for research. The aim of this study is to analyse the use of the Spanish PISA results by the scientific community. A bibliometric analysis of 119 scientific articles included in the Scopus, Eric EBSCOHost and Web of Science databases was carried out between 2002 and 2019 using frequency analysis, MDS analysis and binary logistic regressions. Results reflect a growing interest in the Spanish PISA results from 2005 to the present within university research in the field of Education, to which most of the authors of our sample pool belong. With regard to the use of PISA information, the majority of studies use data for generating knowledge, with socio-economic issues such as the family and equity being the ones with the greatest presence alongside student performance. We conclude that, despite the fact that PISA has been slow to have an impact among the Spanish academic community, its results are increasingly being used as primary source of information for carrying out detailed studies on a wide range of subjects. However, PISA is used generically as an indicator of the quality of the education system, without authors going into detail into the contextualised analysis of performance for the competences under study.
\end{abstract}

Keywords: PISA; information dissemination; norm-referenced evaluation; competences; bibliometric analysis.

\title{
Introducción
}

Las evaluaciones internacionales han pasado a ocupar un lugar privilegiado en el debate sobre políticas educativas (Meyer \& Benavot, 2013). Como ejemplo, el Programa para la Evaluación Internacional de los Estudiantes (PISA) se presentó en 1999 con el objetivo de establecer:

Un compromiso entre los gobiernos de los países de la OCDE para supervisar los resultados de los sistemas educativos en términos de rendimiento de los estudiantes, dentro de un marco común internacionalmente acordado. Si bien se espera que muchas personas de los países participantes, entre ellas profesionales y sociedad en general, utilicen los resultados de la encuesta para una variedad de propósitos, la razón principal para la elaboración y realización de esta evaluación internacional a gran escala tiene por objeto proporcionar una base empírica de información que servirá de base para las decisiones políticas (OECD, 1999, p. 7).

La OCDE ha alcanzado parte de sus objetivos, ya que PISA ha adquirido un importante impacto social y mediático, convirtiéndose en uno de los agentes más activos en el debate sobre políticas educativas y en una forma de medir el potencial educativo y económico de los países participantes (Grek, 2009; Pérez y Soto, 2011; Sellar \& Lingard, 2014).

A pesar de su relevancia, estos estudios no están exentos de limitaciones metodológicas, como la representatividad de la muestra, las tasas de no respuesta o discrepancias interculturales respecto a la traducción, interpretación y comprensión de las preguntas de la prueba (El Masri et al., 2016; Fernández-Cano, 2016; Kankaraš \& Moors, 2014; Rutkowski \& Rutkowski, 2016; Spaull, 2019). 
Asimismo, Rutkowski y Rutkowski (2010) señalan la falta de transparencia como otra de las grandes limitaciones, a pesar de los esfuerzos de sus responsables por hacer públicos sus informes, manuales y bases de datos. La disponibilidad de los informes y de los datos primarios ha facilitado el objetivo pretendido por PISA de que puedan ser utilizados por otros agentes. Por una parte, los medios de comunicación han jugado un papel crucial para trasladar la información generada por los informes a la sociedad, convirtiéndose en "uno de los principales medios de difusión de los resultados e implicaciones que tiene PISA tanto a nivel académico como político y social" (González-Mayorga et al., 2017, p. 3). No obstante, autores como Ferrer y Massot (2005) y Sanz et al. (2020) consideran que el uso que ha hecho la prensa de los resultados de PISA no ha sido el más adecuado, contribuyendo a la formación de una imagen negativa entre la opinión pública.

Además de los medios de comunicación, la comunidad científica ha empleado la información aportada por PISA como fuente para la investigación, gracias a la capacidad que tienen las evaluaciones a gran escala de interrelacionar diferentes temáticas educativas (Leunda et al., 2017). Lindblad et al. (2015) destacan la importancia de la literatura gris en estos estudios (especialmente las notas de prensa e informes publicados por la OCDE), en contraposición con la literatura científica. Sin embargo, diversos estudios ponen de manifiesto la presencia de PISA en las publicaciones académicas. En esta línea, Domínguez et al. (2012) llevaron a cabo un análisis bibliométrico de las publicaciones científicas en los primeros años de PISA (2002-2010), encontrando un total de 322 artículos. En este estudio se concluyó que PISA tuvo un impacto considerable en la investigación científica internacional en sus inicios, sobre todo a partir del año 2007. Asimismo, se comprobó que PISA suscita interés no sólo en el campo de la Educación, sino también de otras ramas como la Psicología, la Economía, las Ciencias o las Matemáticas.

Lindblad et al. (2015) realizaron una revisión sistemática de 59 artículos que establecían comparaciones entre países de los resultados de PISA de 1999 a 2014. En dicho análisis, se determina que los temas más tratados son las diferencias socioculturales, étnicas y de género, por delante del rendimiento $\mathrm{u}$ otras variables intrínsecas como la motivación, los intereses o las expectativas. Hallazgos similares obtuvieron Hopfenbeck et al. (2018), quienes tras el análisis de 654 artículos del intervalo 2002-2014 determinaron que la mayoría emplean los datos de PISA como base científica para generar conocimiento sobre variables socioeconómicas, como el estatus familiar y las desigualdades, y el rendimiento del alumnado.

Como continuación y complemento a estos estudios, el presente trabajo se centra en España como país objeto de estudio de los artículos de investigación relacionados con PISA. Para ello, se ha empleado una metodología similar a la utilizada por Domínguez et al. (2012), cuyo procedimiento se aborda a continuación.

\section{Método}

\section{Objetivos}

Partiendo del objetivo inicial previsto por PISA (OCDE, 1999) sobre la expectativa de que diferentes sectores de la sociedad utilicen sus resultados como base empírica para la toma de decisiones, el objetivo principal de este estudio es analizar qué, cómo 
y con qué propósitos han utilizado los investigadores los resultados de PISA desde sus inicios en 2002 hasta 2019 cuando se analiza España de forma específica. Para ello, se plantean dos objetivos específicos: a) describir la evolución de las publicaciones científicas, en qué revistas, qué autores, con qué países se ha establecido una comparación, cuáles son las competencias analizadas y los temas principales que aborda PISA; y b) describir la asociación de los temas tratados en los artículos analizados en función del uso que se da a los resultados de PISA.

\section{Enfoque metodológico}

El enfoque de este estudio es bibliométrico, cuyo fin es analizar las publicaciones y sus propiedades para obtener información sobre el comportamiento y dinámica de la ciencia (Gingras, 2016). Por su parte, Pérez et al. (2003) diferencian entre bibliometría descriptiva y evaluativa. La bibliometría descriptiva explica de forma general la información producida, analizando, además, su contenido; mientras que la evaluativa mide la incidencia que puedan tener las propiedades de la publicación desde un punto de vista científico y social. En el presente trabajo se empleará el enfoque descriptivo. Este tipo de enfoque es habitual en el ámbito educativo (Archambault y Gagné, 2004; Domínguez et al., 2012; Ramírez-Gutiérrez et al., 2019; Sánchez y Pascual, 2019; Yanniris \& Huang, 2018).

\section{Estrategia de búsqueda y selección de artículos}

Para la selección de los documentos, se realizó una búsqueda inicial en las bases de datos ERIC EBSCOhost, Scopus y Web of Science (core collection). En las tres bases de datos se empleó "PISA" como término de búsqueda en el título, el resumen y las palabras clave. Se utilizó la siguiente sintaxis: en ERIC EBSCOhost TI PISA OR AB PISA OR KW PISA; en Scopus TITLE-ABS-KEY (PISA); y en WOS TI=(PISA) OR AB=(PISA) OR $\mathrm{AK}=(\mathrm{PISA})$. En los tres casos se incluyeron dos criterios adicionales: a) ser artículos de revista; y b) publicados entre enero de 2002 y diciembre de 2019, teniendo en cuenta que la publicación del informe de resultados del primer ciclo de PISA (PISA 2000) se llevó a cabo en diciembre de 2001, y que la del último estudio realizado hasta la fecha (PISA 2018) fue en diciembre de 2019. En este punto se recuperaron 1182 artículos de ERIC EBSCOhost, 5210 de Scopus y 4226 de la colección principal de Web of Science. Se eliminaron los duplicados utilizando el gestor bibliográfico Mendeley.

Posteriormente, se seleccionaron los artículos que cumplían dos criterios de inclusión:

- Hacer referencia a PISA como el Programa Internacional de Evaluación de los Estudiantes y no a otros términos similares, como el "Síndrome de Pisa" o la ciudad de Pisa. Se seleccionaron 1875 artículos.

- Que el país objeto de estudio fuera España de forma específica, descartando 1756 artículos. Entre estos artículos descartados se encuentran aquellos que solo analizan España como un país más dentro de un estudio de diversos países.

Para la aplicación de estos dos criterios fue necesario realizar la lectura de los resúmenes y, cuando fue necesario, del texto completo de los artículos. El corpus final se compone de 119 artículos. 


\section{Variables analizadas}

Una vez conformado el corpus final, se procedió a extraer la información de los artículos. Las siguientes variables se obtuvieron directamente de los artículos:

- Año de publicación.

- Área de la revista. Se empleó el segundo nivel de categorización de Scimago Journal Rank (SJR) denominado category para la clasificación del área de las revistas.

- Apellidos y nombre de los autores.

- Revista.

Por otro lado, la información de las siguientes variables se extrajo a partir del análisis de contenido de los artículos:

Tipo de estudio. Se tomó como referencia la clasificación propuesta por Springer (s.f.), que diferencia entre estudios de investigación original, de revisión y metodológicos, refiriéndose estos últimos a los que analizan las características métricas de PISA.

- País objeto de estudio, aparte de España.

- Competencias: lectora, matemática y científica.

- Temas analizados.

- Estudiantes: hacen referencia al rendimiento académico. Se relaciona de forma recurrente con otros temas incluidos en este estudio e, incluso, con variables de índole psicológica (ansiedad, autoeficacia, motivación, percepción, memoria...).

- Profesorado: está relacionada con la actuación y práctica docente, ratios, formación permanente, estatus social o la relación profesor-alumno, entre otros.

- Familia: se relaciona con las características socioeconómicas del alumnado y antecedentes familiares, así como con el acceso a la cultura en el hogar.

- Género: aborda, principalmente, las diferencias de género (término utilizado en los informes PISA para referirse a las diferencias entre chicos y chicas). Guarda una estrecha relación con variables como la equidad o el rendimiento.

- Equidad: se relaciona con la igualdad de oportunidades.

- Centros educativos: se refiere a las características de los centros (espacios y recursos) y a su titularidad.

- Sistema educativo: hace referencia a la estructura educativa del país y a las políticas educativas.

- Eficiencia, eficacia y calidad: hace referencia a indicadores concretos de evaluación del sistema educativo.

- TICs: hace referencia a la competencia digital del alumnado y del profesorado, o al empleo de programas instruccionales asistidos por ordenador.

- El uso que se da de la información generada por PISA:

- Si se realiza un análisis de los resultados españoles de PISA publicados en los informes.

- Si se emplean las bases de datos españolas de PISA para generar nuevo conocimiento.

- Si menciona a PISA. 


\section{Análisis de los datos}

Inicialmente se creó una base de datos en Excel de los 119 artículos con la información de las variables detalladas anteriormente, tanto las recuperadas directamente, como las que se extrajeron a partir del análisis de contenido utilizando técnicas de análisis cualitativo. Completada la base de datos, se exportó al software IBM SPSS Statistics v26 para proceder al análisis. Se realizaron análisis de frecuencias para la obtención de los resultados del primer objetivo específico. Para analizar la concurrencia de los temas, se calculó una matriz de similaridades utilizando el índice de similaridad de Jaccard $J i j=C i j /(C i+C j-C i j)$ por su simplicidad, facilidad de interpretación y amplio uso. Esta matriz se utilizó para realizar un análisis MDS (escalamiento multidimensional), cuyo resultado ha permitido realizar un mapa de representación en dos dimensiones.

Para el segundo objetivo, describir la asociación de los temas tratados en función del uso que se da a los resultados de PISA, se realizaron pruebas de chi-cuadrado para tablas de $2 \times 2$ y se calculó phi como medida del grado de asociación. No obstante, con el fin de comprobar la interacción entre las variables explicativas, se realizaron análisis de regresión logística binaria. El uso de esta técnica con el objetivo de describir solo la asociación o correlación entre un grupo de variables y una variable dicotómica (y no la predicción) está fundamentado en Jovell (1995) o Silva y Barroso (2004). En concreto, Jovell (1995) señala que "en los estudios en que las características definidas por ambos tipos de variables -independiente y dependientes- se miden en el mismo momento en el tiempo, su relación se interpreta en términos de asociación o correlación" (p.15). Esto es así en todos los estudios de tipo transversal o en los que no se pueda establecer claramente las relaciones de causa-efecto. Algunos estudios incluso utilizan esta técnica en casos en los que los estimadores son expost (Bárcena-Martín et al., 2012).

Los dos tipos de análisis, chi-cuadrado y regresión logística, identificaron las mismas variables y su importancia para describir la relación con la variable criterio, por lo que se ha optado por presentar los resultados de las regresiones logísticas, por su mayor potencia en el análisis de la interacción entre los estimadores.

\section{Resultados}

\section{Evolución en la publicación de artículos}

En los inicios (2002-2004), no se publicó ningún artículo sobre PISA (ver Figura 1). Desde entonces, el volumen de artículos ha aumentado de forma continua, desde un artículo en 2005 hasta los 23 en 2018. El mayor incremento lo podemos encontrar entre 2015 y 2016, pasando de 4 a 15 artículos. Asimismo, se observa que el número de artículos publicados en 2019 ha sido menor respecto al año anterior. 


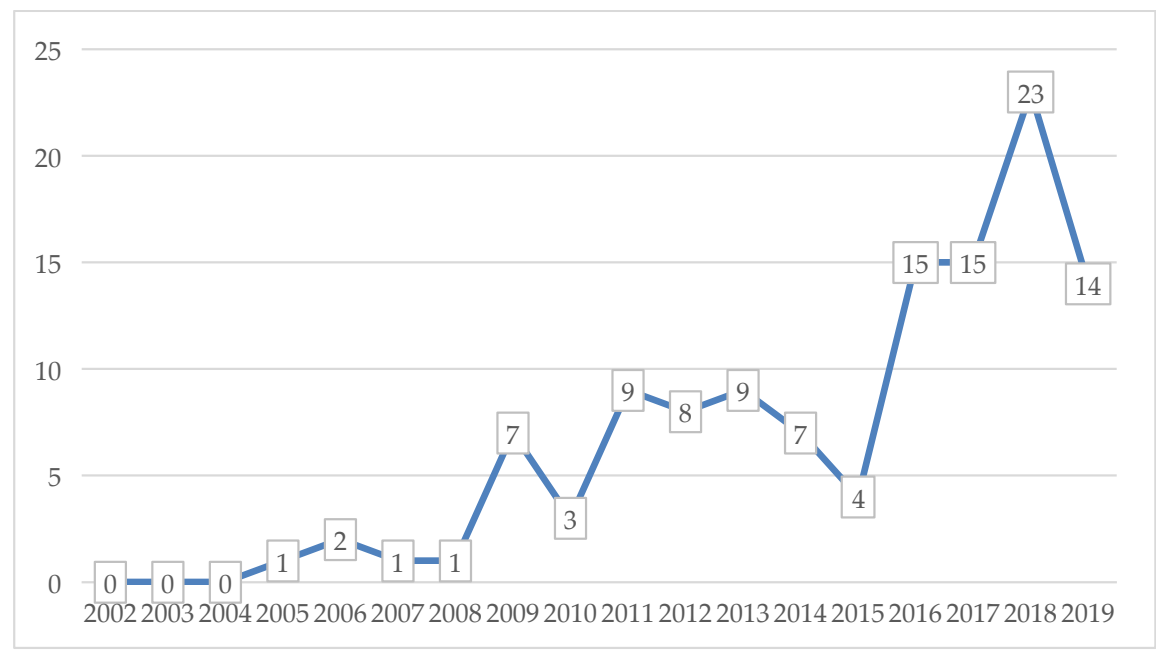

Figura 1. Evolución de las publicaciones.

\section{Revistas y tipo de artículos}

Los 119 artículos han sido publicados en 74 revistas. El idioma de publicación principal fue el castellano, con un 59.7\% (71), seguido del inglés, con un 40.3\% (48), siendo los dos únicos idiomas de publicación utilizados en los artículos analizados.

Respecto al área de las revistas, la Tabla 1 muestra que el 64.7\% de los artículos (77) se han publicado en revistas del área de Educación, seguidas de revistas de Economía, con un $15.1 \%$ (18), de revistas de Psicología, con un 9.2\% (11), y de revistas multidisciplinares de Ciencias Sociales, con un 3.4\% (4) del total.

Tabla 1

Área de las revistas

\section{Frecuencia Porcentaje}

\begin{tabular}{llll} 
& Educación & 77 & 64.7 \\
\cline { 2 - 4 } & Economía y Econometría & 18 & 15.1 \\
\cline { 2 - 4 } & Psicología & 11 & 9.2 \\
\cline { 2 - 4 } Aultidisciplinar de Ciencias Sociales & 4 & 3.4 \\
\cline { 2 - 4 } & Geografía, Planificación y Desarrollo & 2 & 1.7 \\
\cline { 2 - 4 } & Ciencias de la Computación & 2 & 1.7 \\
\cline { 2 - 4 } & Sociología y Ciencias Políticas & 2 & .8 \\
\cline { 2 - 4 } & Artes y Humanidades & 1 & .8 \\
\cline { 2 - 4 } & Lingǘstica y Lenguaje & 1 & 100.0 \\
\cline { 2 - 4 } & Audición y Lenguaje & 119 & \\
\cline { 2 - 4 } & Total & &
\end{tabular}


Por otro lado, respecto a la revista, el 35.3\% (42) de los artículos fueron publicados en ocho revistas, con tres o más publicaciones cada una (Tabla 2). En este sentido, la Revista de Educación es la que ha publicado un mayor número de artículos, con un $16 \%$ (19) del total.

Tabla 2

Revistas que han publicado sobre PISA

\begin{tabular}{lcc}
\hline & Frecuencia & Porcentaje \\
\hline Revista de Educación & 19 & 16.0 \\
\hline Cultura y Educación & 4 & 3.4 \\
\hline Psicothema & 4 & 3.4 \\
\hline Eureka & 3 & 2.5 \\
\hline $\begin{array}{l}\text { Profesorado. Revista de Curriculum y Formación del Profe- } \\
\text { sorado }\end{array}$ & 3 & 2.5 \\
\hline $\begin{array}{l}\text { RELIEVE. Revista Electrónica de Investigación y Evaluación } \\
\text { Educativa }\end{array}$ & 3 & 2.5 \\
\hline Revista Española de Pedagogía & 3 & 2.5 \\
\hline $\begin{array}{l}\text { RIDEP. Revista Iberoamericana de Diagnóstico y Evaluación } \\
\text { Psicológica }\end{array}$ & 3 & 2.5 \\
\hline Otras revistas (66) & 77 & 64.7 \\
\hline Total & 119 & 100.0 \\
\hline
\end{tabular}

En cuanto al tipo de artículos, un $85.7 \%$ (102) corresponde a artículos de investigación, mientras que la presencia de artículos de revisión y metodológicos es mucho menor, con un $10.1 \%$ (12) y un $4.2 \%$ (5) respectivamente.

\section{Autores que han publicado sobre PISA y su afiliación}

El 94.1\% (112) de los artículos fueron publicados por autores universitarios, mientras que el resto son firmados por autores con diferentes afiliaciones, como centros de investigación (3), profesores de Educación Secundaria (2), centros de evaluación (1) y otras entidades (1) (ver Tabla 3).

Tabla 3

Afiliación de los autores

\begin{tabular}{llcc}
\hline & & Frecuencia & Porcentaje \\
\hline \multirow{4}{*}{ Afiliación } & Universidad & 112 & 94.1 \\
\cline { 2 - 4 } & Centro de investigación & 3 & 2.5 \\
\cline { 2 - 4 } & Educación Secundaria & 2 & 1.7 \\
\cline { 2 - 4 } & Centro de evaluación & 1 & 0.8 \\
\cline { 2 - 4 } & Entidad / Asociación & 1 & 0.8 \\
\cline { 2 - 4 } & Total & 119 & 100.0 \\
\hline
\end{tabular}


Respecto a la distribución de los autores, los 119 artículos están firmados por 205 autores diferentes, siendo dos autores los que más publicaciones acumulan, con 8 registros cada uno. Cabe destacar que un 31.3\% (62) del total de autores tienen dos o más autorías, mientras que el porcentaje restante (69.7\%) sólo han publicado un artículo.

\section{Estudios comparados}

De acuerdo con los criterios de selección, la totalidad de los artículos tienen a España como país objeto de estudio. Sin embargo, se encontraron unos pocos artículos en los que se lleva a cabo una comparación de la realidad española con los resultados de otros países, como Alemania (3), Argentina (2), Reino Unido (2), Bélgica (1) y Finlandia (1) (ver Tabla 4). En el caso de Alemania, Argentina y Bélgica, los artículos están firmados por autores con afiliación española y de dichos países de procedencia. Sin embargo, en el caso de Reino Unido y Finlandia, todos los autores tienen sus datos de afiliación de España.

Tabla 4

Países objeto de estudio (respuestas múltiples sobre n=119)

\begin{tabular}{llcc}
\hline & & \multicolumn{3}{c}{ Respuestas } \\
\cline { 2 - 4 } & & Frecuencia & Porcentaje de artículos \\
\hline \multirow{4}{*}{$\begin{array}{l}\text { Países objeto } \\
\text { de estudio }\end{array}$} & España & 119 & 100.0 \\
\cline { 2 - 4 } & Alemania & 3 & 2.5 \\
\cline { 2 - 4 } & Argentina & 2 & 1.7 \\
\cline { 2 - 4 } & Bélgica & 1 & 0.8 \\
\cline { 2 - 4 } & Reino Unido & 2 & 1.7 \\
\cline { 2 - 4 } & Finlandia & 1 & 0.8 \\
\hline Total & & 128 & \\
\hline
\end{tabular}

a. Grupo de dicotomía tabulado en el valor 1.

\section{Contenido de los artículos}

En este apartado se presentan los resultados sobre el contenido de los artículos, diferenciando entre las competencias principales de PISA y las variables que hacen referencia a temas educativos (estudiantes, familia, equidad, centros educativos, sistema educativo, profesorado, eficiencia y TIC).

Teniendo en cuenta las competencias (lectora, matemática y científica), casi la mitad de los artículos (48.7\%) no analizan ninguna de estas, no pudiéndose observar una tendencia clara en la evolución por años con respecto a los artículos que sí las analizan (Figura 2). 


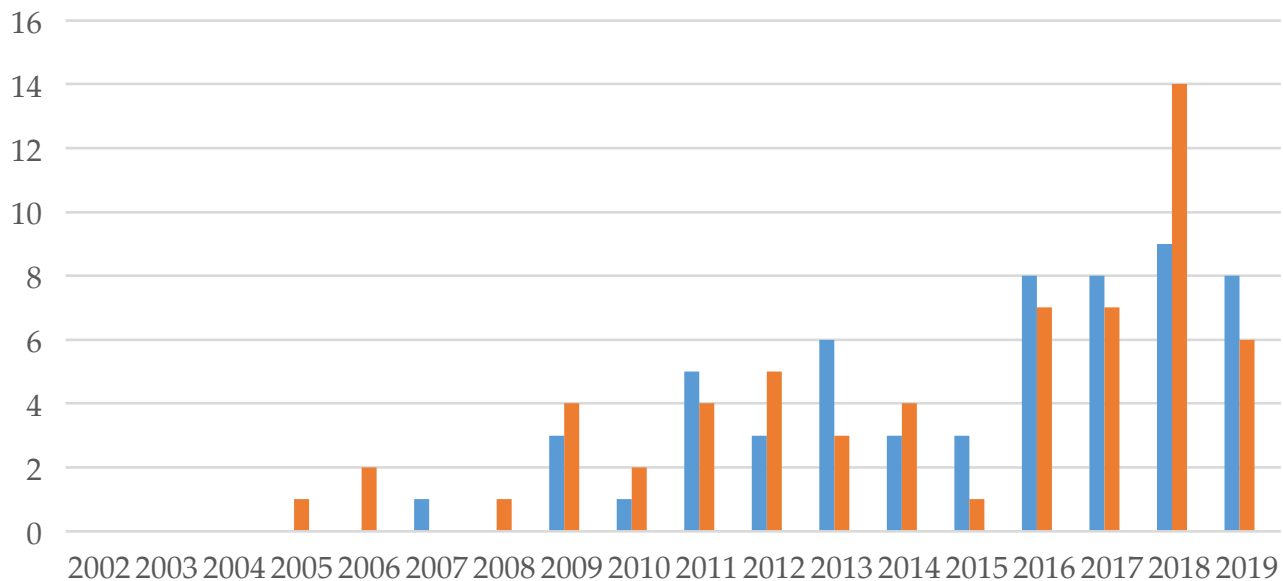

Figura 2. Evolución por años de los artículos según analicen o no las competencias PISA.

Por otro lado, de los 61 artículos que analizan competencias, 16 lo hacen de forma exclusiva sobre la competencia lectora, 16 sobre la científica y 10 sobre la matemática (ver Figura 3). Asimismo, 19 de estos artículos lo hace de las tres competencias de forma simultánea, mientras que no se han encontrado artículos que combinen dos de las tres competencias (Lectora-Matemática, Lectora-Científica o Matemática-Científica). En conjunto, la competencia lectora y la científica son las más estudiadas, con un $57.4 \%(16+19)$ de los casos, mientras que la competencia matemática es analizada en un $47.5 \%(10+19)$.

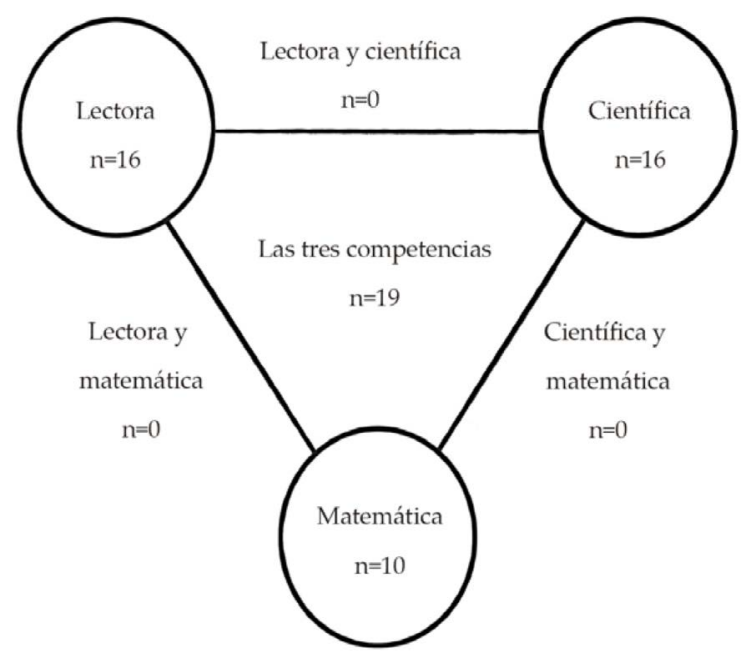

Figura 3. Artículos que analizan competencias. 
Por último, respecto a los temas tratados, como era de esperar, el rendimiento de los estudiantes es el aspecto más analizado (76.5\%), seguido, con un porcentaje bastante menor, por: la familia, atendiendo a características socioeconómicas y antecedentes familiares (35.3\%); la equidad, haciendo referencia a la igualdad de oportunidades (31.9\%); los centros educativos, sus características y titularidad (29.4\%); el sistema educativo, con el efecto de las políticas educativas (28.6\%); el desempeño del profesorado y su práctica docente $(21.8 \%)$; y la eficiencia, eficacia y calidad como indicadores concretos de evaluación del sistema educativo (17.6\%). Por otro lado, los temas menos abordados son la perspectiva de género y el uso de las TIC, con un $9.2 \%$ y un $5.9 \%$ respectivamente (ver Tabla 5).

Tabla 5

Temas analizados (respuestas múltiples sobre $n=119$ )

\begin{tabular}{|c|c|c|c|}
\hline & & \multicolumn{2}{|c|}{ Respuestas } \\
\hline & & Frecuencia & Porcentaje de artículos \\
\hline \multirow{9}{*}{ Temas $^{\mathrm{a}}$} & Estudiantes & 91 & 76.5 \\
\hline & Familia & 42 & 35.3 \\
\hline & Equidad & 38 & 31.9 \\
\hline & Centros educativos & 35 & 29.4 \\
\hline & Sistema educativo & 34 & 28.6 \\
\hline & Profesorado & 26 & 21.8 \\
\hline & Eficiencia, eficacia y calidad & 21 & 17.6 \\
\hline & Género & 11 & 9.2 \\
\hline & TIC & 7 & 5.9 \\
\hline Total & & 305 & \\
\hline
\end{tabular}

a. Grupo de dicotomía tabulado en el valor 1.

En la Figura 4 se puede observar el resultado del análisis MDS basado en la matriz de similaridades calculada con el índice de Jaccard. El índice de stress bruto normalizado resultante para dos dimensiones fue muy bajo (0.041), lo que permite interpretar el mapa bidimensional más fácilmente. Como puede observarse, los temas más tratados están próximos (Estudiantes, Centros Educativos, Equidad y Familia), mientras que los demás no aparecen relacionados de forma clara, especialmente Género y TIC, los cuales aparecen como temas que se tratan de forma independiente. 


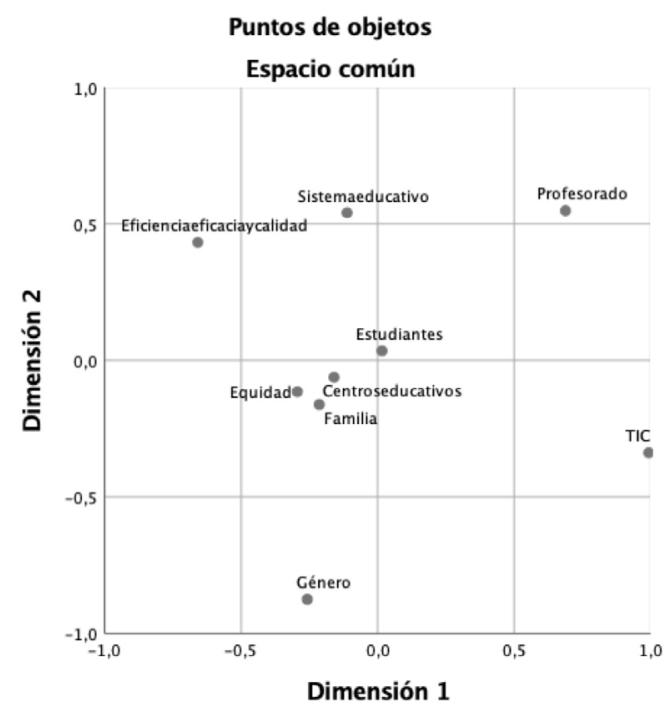

Figura 4. Mapa de proximidad de los temas analizados por PISA.

\section{Uso de la información generada por PISA}

Respecto al segundo objetivo sobre el uso de la información generada por PISA, se puede observar que, en más de la mitad de los artículos (54.6\%), los autores hacen un aprovechamiento de las bases de datos de PISA con el objetivo de generar nuevo conocimiento sobre temas diversos (género, familia, equidad, etc.), mientras que un 28.6\% (34) analizan los resultados publicados en los informes españoles de PISA. Por otro lado, un $16.8 \%$ (20) de los artículos mencionan PISA sin hacer uso de sus bases de datos ni llevar a cabo un análisis de sus resultados (Tabla 6).

Tabla 6

Uso de la información generada por PISA

\begin{tabular}{llccc}
\hline & & Frecuencia & Porcentaje \\
\hline \multirow{3}{*}{ Uso de PISA } & Utilizan bases de datos & 65 & 54.6 \\
\cline { 2 - 5 } & Analizan resultados de informes PISA & 34 & 28.6 \\
\cline { 2 - 4 } & Mencionan PISA & 20 & 16.8 \\
\cline { 2 - 4 } & Total & 119 & 100.0 \\
\hline
\end{tabular}

Para determinar la asociación de los temas abordados en los artículos y el uso que se hace de PISA se llevaron a cabo regresiones logísticas binarias, con el enfoque mencionado en el apartado de método. Se describen los resultados de los modelos estadísticamente significativos. 
En primer lugar, se examina la relación entre los temas analizados y los artículos que utilizan bases de PISA. Las pruebas ómnibus de coeficiente del modelo de regresión determinaron que éste fue estadísticamente significativo $\left(\chi^{2}=20.015, \mathrm{gl}=9, \mathrm{p}<.018\right)$. Asimismo, el modelo explicó el $20.7 \%$ de la varianza de los artículos que analizan PISA (Nagelkerke R2=.207) y clasificó correctamente el 52.9\% de los casos. Como se observa en la Tabla 7 , sólo resultó significativa la variable familia $(p<.05)$, significando que el uso de las bases de datos de PISA se relaciona más con el análisis de este tema que con los otros.

Tabla 7

Influencia de los temas analizados en los artículos que utilizan bases de datos de PISA

\begin{tabular}{lccccccc}
\multicolumn{1}{c}{ Temas } & $\boldsymbol{\beta}$ & $\begin{array}{c}\text { Error } \\
\text { estándar }\end{array}$ & Wald & gl & $\mathbf{p}$ & $\exp (\boldsymbol{\beta})$ \\
\hline Estudiantes & .377 & .501 & .566 & 1 & .452 & 1.458 \\
\hline Profesorado & .169 & .531 & .101 & 1 & .751 & 1.184 \\
\hline Eficiencia, eficacia y calidad & -.844 & .551 & 2.341 & 1 & .126 & .430 \\
\hline Equidad & -.595 & .586 & 1.033 & 1 & .309 & .551 \\
\hline Género & -.025 & .759 & .001 & 1 & .974 & .975 \\
\hline Familia & 1.925 & .638 & 9.092 & 1 & .003 & 6.855 \\
\hline Sistema educativo & -.597 & .471 & 1.609 & 1 & .205 & .550 \\
\hline Centros educativos & -.612 & .584 & 1.100 & 1 & .294 & .542 \\
\hline TIC & .520 & .918 & .321 & 1 & .571 & 1.682 \\
\hline
\end{tabular}

Para la relación entre los temas y los artículos que analizan los resultados de PISA también se determinó que el modelo era significativo $\left(\chi^{2}=23.539, \mathrm{gl}=9, \mathrm{p}<.005\right.$; Nagelkerke R2=.256), clasificando correctamente el $75.6 \%$ de los casos. En este modelo, se observó que dos variables, estudiantes $(\exp (\beta)=0.303)$ y familia $(\exp (\beta)=0.130)$, fueron estadísticamente significativas, pero de signo negativo, significando que estos dos temas tienen menos probabilidad que el resto de ser tratados cuando se analizan los resultados de PISA (Tabla 8).

Tabla 8

Influencia de los temas analizados en los artículos que analizan resultados de PISA

\begin{tabular}{lcccccc}
\multicolumn{1}{c}{ Temas } & $\boldsymbol{\beta}$ & Error estándar & Wald & gl & $\mathbf{p}$ & $\exp (\boldsymbol{\beta})$ \\
\hline Estudiantes & -1.195 & .542 & 4.868 & 1 & .027 & .303 \\
\hline Profesorado & -.813 & .654 & 1.547 & 1 & .214 & .443 \\
\hline Eficiencia, eficacia y calidad & .269 & .584 & .213 & 1 & .644 & 1.309 \\
\hline Equidad & .777 & .633 & 1.507 & 1 & .220 & 2.175 \\
\hline Género & 1.136 & .806 & 1.985 & 1 & .159 & 3.114 \\
\hline Familia & -2.038 & .731 & 7.778 & 1 & .005 & .130 \\
\hline
\end{tabular}




\begin{tabular}{lcccccc}
\multicolumn{1}{c}{ Temas } & $\boldsymbol{\beta}$ & Error estándar & Wald & gl & $\mathbf{p}$ & $\exp (\boldsymbol{\beta})$ \\
\hline Sistema educativo & .340 & .493 & .475 & 1 & .491 & 1.405 \\
\hline Centros educativos & 1.206 & .650 & 3.446 & 1 & .063 & 3.340 \\
\hline TIC & -19.623 & 14857.585 & .000 & 1 & .999 & .000 \\
\hline
\end{tabular}

A continuación, se comprobó la relación entre los temas analizados y los artículos que hacen una referencia secundaria a PISA, determinando que el modelo no era significativo tanto en las pruebas ómnibus $\left(\chi^{2}=14.264, \mathrm{gl}=9, \mathrm{p}<.113\right)$ como en la prueba Hosmer y Lemeshow $\left(\chi^{2}=3.650, \mathrm{gl}=7, \mathrm{p}<.819\right)$.

Por último, se analizó la relación entre los temas tratados y los artículos que estudian alguna de las competencias principales de PISA, siendo un modelo válido $\left(\chi^{2}=35.183\right.$, $\mathrm{gl}=9, \mathrm{p}<.000$; Nagelkerke $\mathrm{R} 2=.341$ ) y con una clasificación correcta del $73.9 \%$ de los casos. En este modelo fueron cuatro las variables significativas. El modelo determinó que un artículo que analiza las competencias de PISA tiene más probabilidades de tratar el tema del género $(\exp (\beta)=10.506)$ y los estudiantes $(\exp (\beta)=5.907)$. Sin embargo, los temas de familia $(\exp (\beta)=.137)$ y sistema educativo $(\exp (\beta)=.232)$ se asocian de forma negativa. Es decir, cuando se analizan resultados de PISA no se hace para tratar los temas de familia y sistema educativo (Tabla 9).

Tabla 9

Influencia de los temas analizados en los artículos que analizan competencias

\begin{tabular}{lcccccc}
\multicolumn{1}{c}{ Temas } & $\boldsymbol{\beta}$ & Error estándar & Wald & gl & $\mathbf{p}$ & $\exp (\boldsymbol{\beta})$ \\
\hline Estudiantes & 1.776 & .564 & 9.915 & 1 & .002 & 5.907 \\
\hline Profesorado & .078 & .575 & .018 & 1 & .893 & 1.081 \\
\hline Eficiencia, eficacia y calidad & -.298 & .576 & .268 & 1 & .605 & .742 \\
\hline Equidad & .104 & .576 & .033 & 1 & .857 & 1.109 \\
\hline Género & 2.352 & .970 & 5.884 & 1 & .015 & 10.506 \\
\hline Familia & -1.987 & .629 & 9.986 & 1 & .002 & .137 \\
\hline Sistema educativo & -1.460 & .527 & 7.686 & 1 & .006 & .232 \\
\hline Centros educativos & .438 & .580 & .570 & 1 & .450 & 1.549 \\
\hline TIC & .000 & .882 & .000 & 1 & 1.000 & 1.000 \\
\hline
\end{tabular}

En síntesis, se observa disparidad en el uso dado por los autores a la información generada por PISA cuando se analiza España de forma específica. En primer lugar, en más de la mitad de los artículos se utilizan las bases de datos de PISA como fuente de información para otros estudios de temas específicos, fundamentalmente la familia. Por tanto, se cumple el objetivo inicial previsto por el proyecto PISA de que se utilicen sus datos como base empírica de otros estudios. En segundo lugar, un tercio de los artículos, realizan un análisis de los resultados de los informes, sin acudir a las bases de datos disponibles. En este caso, el foco de estudio es el rendimiento de los estudiantes en una o todas las competencias analizando la variable género. Por último, destaca otro conjunto de artículos que, aunque inferior, supone un $17 \%$ del total en los que 
PISA se menciona sin profundizar en sus resultados para referirse de forma genérica a la calidad del sistema educativo.

\section{Discusión y conclusiones}

El propósito de este estudio es determinar cómo se ha utilizado la información generada por los resultados españoles de PISA en el ámbito académico.

Respecto al primer objetivo, en primer lugar, se puede afirmar que PISA tardó más de 7 años en impactar en el ámbito académico español. Sin embargo, se ha producido un fuerte crecimiento en 2016, que parece indicar que este interés se mantendrá.

En segundo lugar, se concluye que los autores son, mayoritariamente, del ámbito universitario, siendo muy escasa la participación de autores de otros ámbitos. Estos autores son, sin embargo, numerosos y de diversas instituciones, lo que indica que el impacto es amplio. Sin embargo, la concentración de estos estudios en autores universitarios no respalda la importancia de la colaboración entre diferentes contextos educativos, especialmente entre los teóricos (universidad e investigación) y los prácticos (profesores de aula y centros educativos) con el objetivo de, por un lado, poner en práctica los hallazgos fruto de la investigación, y, por otro, favorecer la reflexión y el autoanálisis de la práctica (Monereo, 2009).

En tercer lugar, se concluye que la mayoría de los estudios se concentran en revistas del campo de la Educación, la Economía y la Psicología. Sin embargo, el interés se ha producido también en otros campos no solo de las ciencias sociales. Al igual que con los autores, los estudios se han publicado en numerosas revistas, lo que vuelve a indicar el amplio impacto de PISA.

En cuarto lugar, respecto al contenido de los artículos, llama la atención que más de la mitad no centran su análisis en ninguna de las competencias objeto de estudio de PISA, (Ministerio de Educación y Formación Profesional, 2019). Esto se debe al interés de los autores en utilizar los datos provenientes de PISA como indicadores generales para fundamentar o ilustrar el análisis de otros aspectos como la segregación escolar, el perfil de los alumnos repetidores o la elección de centro (Arroyo et al., 2019; Murillo et al., 2017; Salinas y Santín, 2012). Por otra parte, respecto a los artículos que mencionan PISA sin analizar sus datos, destaca que lo suelen hacer para describir con la mención a PISA, la calidad del sistema educativo en su conjunto, a modo de introducción para su investigación.

En quinto lugar, la mayor parte de los artículos que analizan alguna de las competencias hacen referencia a las tres (lectora, matemática y científica), mientras que, a título individual, son la lectura y la competencia científica las que suscitan mayor interés. Estos resultados contrastan con los de Domínguez et al. (2012), cuyo estudio establece que la competencia matemática fue la más analizada entre 2002 y 2010 . Así pues, se pone de manifiesto cómo la competencia científica y la lectura han ganado protagonismo en la última década, auspiciados por un mayor apogeo de conceptos como las enseñanzas STEM (Science, Technology, Engineering, Mathematics) o la lectura digital, introducida en 2009 bajo la denominación PISA-ERA (Electronic Reading Assessment) (Gil-Flores et al., 2012; Kusumastuti et al., 2019; López et al., 2019; Ministerio de Educación, 2011; Stoller \& Nguyen, 2020; Vázquez-Cano, 2017). 
Por último, respecto a los temas tratados, el más abordado es, como era de esperar, el rendimiento académico, variable dependiente principal de PISA, seguido de temas próximos como centros educativos, equidad y familia. Otros temas menos tratados como género y TIC, se tratan de forma independiente. Esta diversidad de temas evidencia el potencial que tiene PISA como fuente de información para la investigación sobre las variables de input, contexto y proceso (Arroyo et al., 2019).

Respecto al segundo objetivo, en primer lugar, se observa que la mayoría de los artículos emplean los datos de PISA sin hacer análisis más detallados, pero cuando se usan, tratan principalmente temas relacionados con la familia, coincidiendo con Hopfenbeck et al. (2018).

En segundo lugar, se observa que las publicaciones que hacen referencia secundaria a PISA no se asocian de forma clara con ninguno de los temas analizados, empleándose la evaluación PISA como justificación para el tratamiento de temas diversos como el inferior desempeño de los estudiantes españoles con respecto a la media de la OCDE, el análisis del fracaso escolar y de la desigualdad social entre Comunidades Autónomas, o las reformas educativas (Cabrera, 2013; López-Yáñez y Sánchez-Moreno, 2013; Pérez et al., 2011; Pérez-Esparrells y Morales, 2012; Salmerón et al., 2018; Santos et al., 2016; Saura y Luego, 2015; Vázquez-Cano, 2017; Ventura, 2017).

Por último, los artículos que analizan competencias guardan una estrecha relación con el género, considerado como uno de los temas que más auge ha tenido no sólo en el campo de las Ciencias Sociales (Fuentes y Renobell, 2020; González de San Román y De la Rica, 2016; Torrecilla et al., 2019).

En síntesis, el proyecto PISA de la OCDE ha tardado en tener impacto en la comunidad académica española, pero suscita un interés amplio, creciente y promueve estudios cada vez más detallados. Podemos decir que el proyecto PISA ha dejado de ser un fin en sí mismo dentro del ámbito académico para convertirse en una valiosa fuente de información para los investigadores en la generación de nuevo conocimiento. Sin embargo, PISA se ha convertido también en un indicador genérico de la calidad del sistema educativo, que en muchas ocasiones se utiliza ya como axioma para fundamentar otro tipo de análisis, ignorando tanto el principal objetivo (el análisis de un reducido número de competencias) como el elevado número de variables independientes disponibles para diferenciar los contextos en los que necesariamente debe enmarcarse el análisis del rendimiento.

La limitación de este estudio es que analiza el impacto de PISA en la academia solo por medio de los artículos de investigación, aunque podría estar impactando de otras formas, lo que requeriría de análisis basados en entrevistas o encuestas. No tanto como limitaciones, sino como futuros estudios, sería interesante comparar la situación descrita en este estudio con lo que ha sucedido en otros países, confirmar el posible techo detectado en el último trienio y, por último, analizar si la investigación académica, más allá de los informes de PISA, ha tenido efecto en las políticas educativas. 


\section{Referencias}

Archambault, E., \& Gagné, E. V. (2004). The use of bibliometrics in the Social Sciences and Humanities. Science-Matrix.

Arroyo, D., Constante, I. A., \& Asensio, I. (2019). La repetición de curso a debate: un estudio empírico a partir de PISA 2015. Educación XX1, 22(2), 69-92. https://doi. org/10.5944/educXX1.22479

Bárcena-Martín, E., Budría, S., \& Moro-Egido, A.I. (2012). Skill mismatches and wages among European university graduates. Applied Economics Letters, 19(15), 1471-1475. https://doi.org/10.1080/13504851.2011.633886

Cabrera, L. J. (2013). Desigualdad social, rendimiento y logro educativos en España (1990-2012): Los desequilibrios regionales aumentan. Revista de Estudios Regionales, 98, 15-49.

Domínguez, M., Vieira, M. J., \& Vidal, J. (2012). The impact of the Programme for International Student Assessment on academic journals. Assessment in Education: Principles, Policy \& Practice, 19(4), 393- 409. https://doi.org/10.1080/0969594X.2012.659175

El Masri, Y. H., Baird, J.-A., \& Graesser, A. (2016). Language effects in international testing: The case of PISA 2006 science items. Assessment in Education: Principles, Policy $\mathcal{E}$ Practice, 23(4), 427-455. https://doi.org/10.1080/0969594X.2016.1218323

Ferrer, F., \& Massot, M. (2005). El proyecto PISA en los medios de comunicación escrita: de la simplificación a la manipulación. Organización y Gestión Educativa, 13(1), 19-22.

Fernández-Cano, A. (2016). Una crítica metodológica de las evaluaciones PISA. RELIEVE, 22(1), art. M15. https://doi.org/10.7203/relieve.22.1.8806

Fuentes, S., \& Renobell, V. (2020). La influencia del género en el aprendizaje matemático en España. Evidencias desde PISA. Revista de Sociología de la Educación-RASE, 13(1), 63-80. https://doi.org/10.7203/RASE.13.1.16042.

Gil-Flores, J., Torres-Gordillo, J. J., \& Perera-Rodríguez, V. H. (2012). The role of online reader experience in explaining students' performance in digital reading. Computers $\mathcal{E}$ Education, 59, 653-660. https://doi.org/10.1016/j.compedu.2012.03.014

Gingras, Y. (2016). Bibliometrics and Research Evaluation: Uses and Abuses. MIT.

González de San Román, A., \& De la Rica, S. (2016). Gender gaps in PISA test scores: the impact of social norms and the mother's transmission of role attitudes. Estudios de Economía Aplicada, 34(1), 79-108.

González-Mayorga, H., Vidal, J., \& Vieira, M.-J. (2017). El impacto del Informe PISA en la sociedad española: el caso de la prensa escrita. RELIEVE, 23(1), art 3. http:// doi.org/10.7203/relieve.23.1.9015

Grek, S. (2009). Governing by the numbers: The PISA "effect" in Europe. Journal of Education Policy, 24(1), 23-37. https://doi.org/10.1080/02680930802412669

Hopfenbeck, T. N., Lenkeit, J., El Masri, Y., Cantrell, K., Ryan, J., \& Baird, J.-A. (2018). Lessons learned from PISA: A systematic review of peer-reviewed articles on the Programme for International Student Assessment. Scandinavian Journal of Educational Research, 62(3), 333-353. https://doi.org/10.1080/00313831.2016.1258726

Jovell, A. J. (1995). Análisis de regresión logística. Centro de Investigaciones Sociológicas. 
Kankaraš, M., \& Moors, G. (2014). Analysis of cross-cultural comparability of PISA 2009 scores. Journal of Cross-Cultural Psychology, 45(3), 381-399. https://doi. org/10.1177/0022022113511297

Kusumastuti, F. A., Rombot, O., \& Ariesta, F. W. (2019). The effect of STEM integration on primary school students' scientific literacy. International Journal of Scientific $\mathcal{E}$ Technology Research, 8(12), 1551-1553.

Leunda, I., Garmendia, I., \& Etxeberria, J. (2017). Statictical Matching en la práctica - Una aplicación a la evaluación del Sistema educativo mediante PISA y TALIS. Revista de Investigación Educativa, 35(2), 371-388. http://dx.doi.org/10.6018/rie.35.2.262171

Lindblad, S., Pettersson, D., \& Popkewitz, T. S. (2015). International comparisons of school results: a systematic review of research on large scale assessments in education. Swedish Research Council.

López, F., García, I., \& Expósito, E. (2019). Rendimiento en ciencias, concepciones epistémicas y vocaciones STEM en las comunidades autónomas españolas. Evidencias desde PISA 2015, políticas y prácticas de mejora. Revista Española de Pedagogía, 77(272), 5-27. https://doi.org/10.22550/REP77-1-2019-09

López-Yáñez, J., \& Sánchez-Moreno, M. (2013). Levers for sustainable improvement of Spanish schools in challenging contexts. Journal of Educational Change, 14, 203-232. https://doi.org/10.1007/s10833-012-9198-x

Meyer, H.-D., \& Benavot, A. (2013). PISA and the Globalization of Education Governance: some puzles and problems. In H.-D. Meyer \& A. Benavot (Eds.), PISA, Power, and Policy: the emergence of global education governance (9-26). Symposium Books.

Ministerio de Educación (2011). PISA - ERA 2009. Programa para la Evaluación Internacional de los Alumnos. Informe español. Secretaría de Estado de Educación y Formación Profesional.

Ministerio de Educación y Formación Profesional (2019). PISA 2018. Programa para la Evaluación Internacional de los Estudiantes. Informe Español. Secretaría General Técnica.

Monereo, C. (2009). PISA como excusa. Repensar la evaluación para cambiar la enseñanza. Graó.

Murillo, F. J., Martínez-Garrido, C., \& Belavi, G. (2017). Segregación escolar por origen nacional en España. OBETS. Revista de Ciencias Sociales, 12(2), 395-423. https://doi. org/10.14198/OBETS2017.12.2.04

OECD (1999). Measuring students' knowledge and skills. A New Framework for Assessment. OCDE Publications Service. https://www.oecd.org/education/school/programmeforinternationalstudentassessmentpisa/33693997.pdf

Pérez, G., Anta, C., Badera, S., García, J., Pérez, M., \& Sarrate, M. (2003). Análisis Bibliométrico en Educación. Incidencia en la calidad universitaria. Ministerio de Educación y Ciencia de España.

Pérez, E., Serrano, S., \& Vico, S. (2011). Cómo mejorar la comprensión lectora a partir de estrategias de aprendizaje en alumnado de $6^{\circ}$ de Primaria. Boletín de AELFA, 11(3), 68-72. https://doi.org/10.1016/s1137-8174(11)70048-6

Pérez, A. I., \& Soto, E. (2011). Luces y sombras de PISA. Sentido educativo de las evaluaciones externas. Cultura y Educación, 23(2), 171-182. https://doi. org/10.1174/113564011795944758 
Pérez-Esparrells, C., \& Morales, S. (2012). El fracaso escolar en España: un análisis por Comunidades Autónomas. Revista de Estudios Regionales, 94, 39-69.

Ramírez-Gutiérrez, Z., Barrachina-Palanca, M., \& Ripoll-Feliu, V. M. (2019). Divulgación de rankings universitarios y eficiencia en educación superior. Análisis bibliométrico y sistémico. Revista de Educación, 384, 255-297. https://doi.org/10.4438/1988592X-RE-2019-384-412

Rutkowski, L., \& Rutkowski, D. (2010). Getting it 'better': The importance of improving background questionnaires in international large-scale assessment. Journal of Curriculum Studies, 42(3), 411-30. http://doi.org/10.1080/00220272.2010.487546

Rutkowski, L., \& Rutkowski, D. (2016). A call for a more measured approach to reporting and interpreting PISA results. Educational Researcher, 45(4), 252-257. https://doi. org/10.3102/0013189X16649961

Salinas, J., \& Santín, D. (2012). Selección escolar y efectos de la inmigración sobre los resultados académicos españoles en PISA 2006. Revista de Educación, 358, 382-405. https://doi.org/10-4438/1988-592X-RE-2010-358-083

Salmerón, L., García, A., \& Vidal-Abarca, E. (2018). WebLEC: a test to assess adolescents' Internet reading literacy skills. Psicothema, 30(4), 388-394. https://doi.org/10.7334/ psicothema2017.395

Sánchez, S., \& Pascual, M. Á. (2019). Análisis bibliométrico de la investigación educativa sobre desventaja sociocultural/socieducativa en el periodo 2015 a 2019. Enseñanza $\mathcal{E}$ Teaching, 37(2), 147-164.

Santos, M. Á., Godás, A., Ferraces, M. J., \& Lorenzo, M. (2016). Academic performance of native and immigrant students: A study focused on the perception of family support and control, school satisfaction, and learning environment. Frontiers in Psychology, 7, 1-12. https://doi.org/10.3389/fpsyg.2016.01560

Sanz, R., Serrano, Á., \& González, A. (2020). PISA: el precio pedagógico de una evaluación internacional. REICE. Revista Electrónica de Investigación Educativa, 22, e22, 1-13. https://doi.org/10.24320/redie.2020.22.e22.2673

Saura, G., \& Luego, J. (2015). Política global más allá de lo nacional. Reforma educativa (LOMCE) y el régimen de estandarización (OCDE). Bordón, 67(1), 135-148. https:// doi.org/10.13042/Bordon.2015.67109

Sellar, S., \& Lingard, B. (2014). The OECD ad the expansion of PISA: New global modes of governance in education. British Educational Research Journal, 40(6), 917-936. https:// doi.org/10.1002/berj.3120

Silva, L. C. \& Barroso, I. M. (2004). Regresión Logística. La Muralla.

Spaull, N. (2019). Who makes it into PISA? Understanding the impact of PISA sample eligibility using Turkey as a case study (PISA 2003-PISA 2012). Assessment in Education: Principles, Policy \& Practice, 26(4), 397-421. https://doi.org/10.1080/09695 94X.2018.1504742

Springer (s.f.). Tipos de artículos de revistas. Springer. https://www.springer.com/la/ authors-editors/tutoriales-de-autores-y-revisores/writing-a-journal-manuscript/ types-of-journal-articles/12022874

Stoller, F. L., \& Nguyen, L. T. H. (2020). Reading habits of Vietnamese University English majors. Journal of English for Academic Purposes, 48, 1-17. https://doi.org/10.1016/j. jeap.2020.100906 
Torrecilla, E. M., Olmos, S., \& Martínez, F. (2019). Explanatory factors as predictors of academic achievement in PISA tests. An analysis of the moderating effect of gender. International Journal of Educational Research, 96, 111-119. https://doi.org/10.1016/j. ijer.2019.06.002

Vázquez-Cano, E. (2017). Analysis of difficulties of Spanish teachers to improve students' digital reading competence. A case study within the PISA framework. Pedagogika, 125(1), 175-194. https://doi.org/10.15823/p.2017.13

Ventura, D. (2017). La lectura en FLE por y para el aprendizaje. Cédille, 13, 479-500.

Yanniris, C., \& Huang, Y. S. (2018). Bibliometric evidence point to loci of empirical knowledge production in environmental education. Cogent Education, 5(1), 1-14. https://doi.org/10.1080/2331186X.2018.1542961

Fecha de recepción: 19 de octubre de 2020.

Fecha de revisión: 8 de noviembre de 2020.

Fecha de aceptación: 29 de abril de 2021. 